\title{
A pilot observational study of the association between fatigue after stroke and C-reactive protein
}

\author{
${ }^{1} \mathrm{~F}$ McKechnie, ${ }^{2} \mathrm{~S}$ Lewis, ${ }^{3} \mathrm{G}$ Mead \\ ${ }^{1}$ Medical Student; ${ }^{2}$ Research Fellow in Geriatric Medicine; ${ }^{3}$ Reader in Geriatric Medicine, University of Edinburgh, UK
}

\section{ABSTRACT}

Background: The aetiology of fatigue after stroke is unknown. We explored the relationship between fatigue and C-reactive protein (CRP) as a marker of inflammation.

Methods: This cross-sectional study recruited inpatients with a stroke (onset within the previous three months) over a five-week period. Those with dysphasia or confusion severe enough to prevent informed consent and those with current infection were excluded. A semi-structured interview determined a) fulfilment of a case definition for fatigue and b) severity of fatigue (fatigue assessment scale, FAS). Venous blood was taken for CRP. A hospital anxiety and depression score (HADS) was used to screen for emotional distress.

Results: Of the 28 patients recruited (mean age 72.7 years, proportion men $47 \%$ ), I5 $(53 \%)$ fulfilled the case definition for fatigue. C-reactive protein data were logarithmically transformed for analysis. C-reactive protein levels did not differ significantly between those with and without fatigue, according to the case definition $(n=28, p=0.35)$. After exclusion of those with pre-stroke fatigue and those with high scores on the HADS (suggestive of emotional distress), the geometric mean CRP of the fatigued group was $16.04 \mathrm{mg} / \mathrm{l}(95 \% \mathrm{Cl}: 7.12-36.14)$ compared with $5.16 \mathrm{mg} / \mathrm{l}$ (95\% Cl: 2.7-9.85) in the non-fatigued group $(n=21, p=0.025$, unpaired $t$ test), but the relationship between FAS and CRP was not statistically significant $(r=0.37$, $p=0.098$ ).

Conclusion: This pilot study is the first to demonstrate an association between fatigue after stroke and higher CRP, after excluding patients with pre-stroke fatigue and those with probable mood disorders. If this finding is confirmed in a larger number of patients, it might provide a target for treating fatigue after stroke.

KEYWORDS C-reactive protein, fatigue, inflammation, stroke

DECLARATION OF INTERESTS Dr Gillian Mead is Assistant Editor of The Journal of the Royal College of Physicians of Edinburgh. This article was submitted to the JRCPE's Clinical Editor and has been peer-reviewed in line with usual JRCPE procedures.
Correspondence to G Mead, Room S1642, Royal Infirmary of Edinburgh, Little France Crescent, Edinburgh EHI6 4SA, UK

tel. +44 (0) I 3 I 242648 I e-mail gillian.e.mead@ed.ac.uk

\section{INTRODUCTION}

Stroke is the most common cause of long-term disability in the Western world. Evidence is beginning to emerge that fatigue after stroke is a common problem and that it is distressing to patients. Recent studies have found that between $30-68 \%$ of patients after stroke have fatigue' and one study ${ }^{2}$ found that $40 \%$ of patients rated their fatigue as among the worst of their symptoms following stroke. Fatigue can cause increased difficulty taking part in physical therapy.' Despite the impact of fatigue after stroke, little research has been done in this area.There are many potential causes of fatigue following stroke, such as physical de-conditioning, metabolic co-morbidities or depression, but currently the aetiology of fatigue remains uncertain. ${ }^{3}$ Animal studies have shown an association between pro-inflammatory cytokines and altered behavioural processes called 'sickness behaviour', which include reduced social behaviour, altered sleep patterns and increased sensitivity to pain. ${ }^{4}$ Thus, it is plausible that in humans cytokines may also induce a 'sickness behaviour' which may include symptoms of fatigue.
Fatigue associated with other conditions has been much more extensively studied than post-stroke fatigue. The high prevalence rate and disabling effects of fatigue following cancer chemotherapy have led to much interest in this field. ${ }^{5-7}$ In particular, an association between pro-inflammatory cytokines and fatigue has been demonstrated in several studies. ${ }^{5-7}$ There is also preliminary evidence of an association between fatigue and inflammation following colorectal surgery ${ }^{8}$ and in multiple sclerosis. ${ }^{9}$

After acute stroke, there is evidence of systemic inflammation. C-reactive protein (CRP) levels are higher in patients with more severe strokes, ${ }^{10}$ and higher CRP is associated with worse outcome $\mathrm{I}^{10,11}$ after correcting for stroke severity. The pro-inflammatory cytokine interleukin 6 (IL-6) is released in acute stroke. Interleukin 6 levels correlate with brain infarct volume and outcome ${ }^{12}$ and predict clinical deterioration early after ischaemic stroke. ${ }^{13}$ To our knowledge, the relationship between systemic inflammation after stroke and fatigue has not been studied. 
Because of the increasing amount of evidence implicating inflammation in fatigue in conditions other than stroke, ${ }^{5-9}$ our aim was to collect data about the relationship between fatigue after stroke and inflammation (as measured by CRP) in a small group of patients with stroke, in order to inform power calculations for a larger study.

\section{METHODS}

\section{Recruitment and initial information gathered}

Patients were recruited between 23 March 2009 and 30 April 2009. Any inpatient on one of three stroke units was potentially eligible to participate, providing they had suffered a stroke within the previous three months. Patients known to have an active infection, those with white cell counts of $>10 \times 10^{9} / \mathrm{l}$ and/or currently receiving antibiotics and those with confusion or dysphasia severe enough to impair ability to give informed consent were excluded. Those with known malignancy and those with inflammatory disease such as rheumatoid arthritis were also excluded. The researcher, Fiona McKechnie, was introduced to potentially eligible patients by the medical team. She provided the patients with information about the study, and each patient was given up to 24 hours to consider whether to participate. Demographic data were extracted from the inpatient medical records of consenting patients, including age, date of stroke and pathological type of stroke (haemorrhagic or ischaemic).

\section{Assessment of fatigue}

Firstly, the researcher confirmed that the participants were aware of the meaning of fatigue, asked whether they had experienced fatigue prior to their stroke, and then performed a semi-structured, face-to-face interview to determine whether they fulfilled a case definition for clinically significant fatigue. ${ }^{14}$ The definition of fatigue was: 'Since their stroke the patient has experienced fatigue, a lack of energy or an increased need to rest every day or nearly every day.' To fulfil the case definition for fatigue, patients needed to have fatigue (rather than boredom or reduced motivation) for $>50 \%$ of waking hours, and this fatigue had to have been present every day or nearly every day since their stroke. This fatigue had to have led to difficulty taking part in everyday activities (for inpatients, this included any physical therapy). This definition had been developed based on patients' description of fatigue, and had been tested for reliability. ${ }^{14}$

Patients were then asked to complete the fatigue assessment scale (FAS). This was chosen as it is feasible, valid and reliable in patients who have had a stroke. ${ }^{15}$ The scale consists of ten questions about aspects of fatigue, each rated on a five-point scale from 'never' to 'always'. ${ }^{16}$ This scale was applied to all patients irrespective of whether they fulfilled the case definition for fatigue.

The hospital anxiety and depression score (HADS) was then administered to screen for emotional distress (including depression and anxiety), which might cause fatigue. ${ }^{17}$

\section{Laboratory analyses}

Immediately following the completion of the questionnaires, a venous blood sample was taken. This was sent to the routine biochemistry laboratory, via the usual hospital transport system, for analysis. C-reactive protein was assayed using a Latex-enhanced immunoturbidimetric method on an automated Olympus AU2700 analyser, according to the manufacturer's protocol. The published analytical sensitivity of the assay is $0.14 \mathrm{mg} / \mathrm{l}$, with reported intra-assay and inter-assay coefficients of variation of $3.22 \%$ and $3.8 \%$ respectively at a CRP concentration of $6.6 \mathrm{mg} / \mathrm{l}$.

\section{Statistical analysis}

All data were entered into SPSS Version 13.0. The FAS was normally distributed. C-reactive protein was normally distributed after $\log 10$ transformation. All these distributions were tested using the Shapiro-Wilk test of normality. All analyses were therefore carried out with the $\log 10$ transformed CRP data and the raw FAS data.

An unpaired t-test was used to compare the levels of CRP between patients with and without fatigue (according to the case definition). The relationship between $\log 10$ transformed CRP and the FAS was analysed using Pearson's correlation coefficient.

We included the whole group in our initial analyses, and then excluded those with pre-stroke fatigue and those with high scores on the HADS, suggestive of emotional distress.

As this was a pilot study, we did not perform power calculations; rather, the purpose of the study was to gain data to inform power calculations for a larger study.

\section{RESULTS}

Thirty-one patients were eligible to participate. Of these, three patients declined (two did not want an additional blood sample to be taken, one did not feel physically well enough). Of the 28 patients included, the mean age was 73 years $(S D=14.1)$ and $15(54 \%)$ were female. Twenty-six had had an ischaemic stroke and two a haemorrhagic stroke. The median time between stroke onset and interview was 35 days (range 3-89 days). Four patients stated that they had suffered from fatigue before their stroke. Of the 28 patients included in the study, $15(53 \%)$ fulfilled the case definition for clinically significant fatigue.

The geometric mean CRP was $8.03 \mathrm{mg} / \mathrm{l}(95 \% \mathrm{Cl}: 3.86-$ $16.71)$ in the fatigued group $(n=15)$, compared with $5.16 \mathrm{mg} / \mathrm{l}(95 \% \mathrm{Cl}: 2.7-9.85)$ without fatigue $(n=13)$. This difference was not statistically significant $(p=0.35$, 


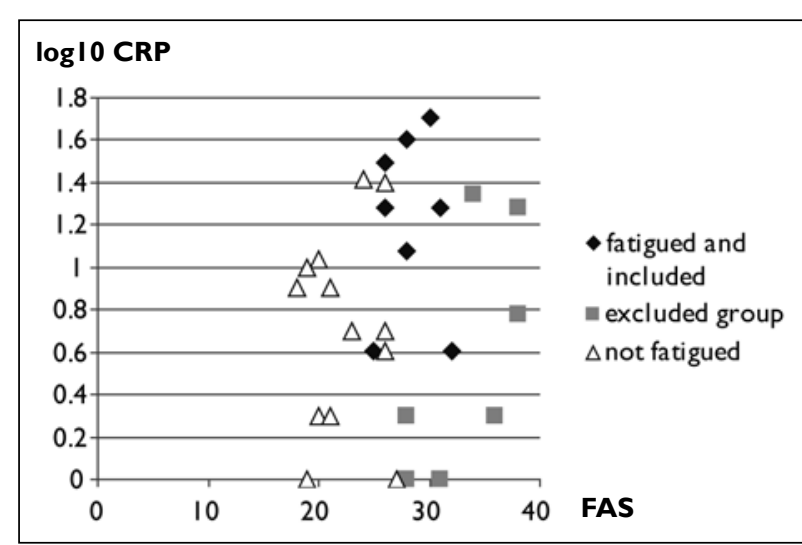

FIGURE I Relationship between fatigue assessment scale and transformed C-reactive protein levels in all 28 patients.

unpaired t-test). There was no significant correlation between FAS and log 10 CRP levels $(r=0.12, p=0.55)$.

We performed further analyses by excluding those four patients who reported pre-stroke fatigue. The geometric mean CRP in the fatigued group $(n=11)$ was $9.43(95 \%$ Cl: 3.98-22.36) compared with 5.16 (95\% Cl: 2.7-9.85) in the non-fatigued group $(n=13, p=0.22)$. There was no significant correlation between $\log 10 \mathrm{CRP}$ and FAS $(r=0.09, p=0.67)$.

We then excluded the three patients who scored highly on the HADS $(28,28$ and 30 respectively), as their fatigue may have been a result of emotional distress. Of the eight remaining patients fulfilling the case definition, the geometric mean CRP was 16.04 (95\% Cl: 7.I236. I4), compared with 5.16 (95\% Cl: 2.7-9.85) in the 13 patients not fulfilling the case definition. This difference between groups was statistically significant $(p=0.025)$. However, there was no significant correlation between $\log 10$ CRP and FAS $(r=0.37, p=0.098)$. Figure I shows the distribution of FAS scores and log 10 CRP levels.

\section{DISCUSSION}

This is the first study to our knowledge to explore the relationship between fatigue and CRP after stroke. Around half of patients fulfilled our case definition for fatigue following stroke, which is similar to the findings of previous studies.' Although CRP levels were higher in patients fulfilling the case definition compared with those who did not, this relationship was not statistically significant. However, when patients with high scores on the HADS (suggestive of mood disorders) and those with pre-stroke fatigue were excluded, CRP levels were significantly higher in patients fulfilling the case definition for fatigue.

This study has a number of limitations. The main one is that the sample size was small. This was a student project so the time to recruit patients was constrained by the time available (5.5 weeks). Secondly, the study focused on inpatients who generally would have had more severe strokes than outpatients, thus limiting the external validity of the results. Thirdly, patients were enrolled at different time points after the onset of their stroke (range 3-89 days). Thus, this study provided only a 'snapshot' of the relationship between fatigue and inflammation and we cannot infer direction of causality; that is, does inflammation cause fatigue or does fatigue cause inflammation?

Additionally, it is known that both fatigue and CRP levels are not stable throughout the first three months following stroke. Future studies should therefore measure CRP at a fixed time point after stroke. Fourthly, because this was a small study, we did not correct for other factors that might be associated with fatigue: for example, a recent study found a relationship between systemic hypotension and fatigue. ${ }^{3}$

Other factors that could influence CRP levels were not recorded. Drugs such as lipid-lowering therapies or angiotensin-converting enzyme inhibitors are commonly used in stroke patients and can affect CRP levels. These factors were not recorded in this study as it was primarily a student project and had been designed to keep analysis as simple as possible. Future studies should take into account the effect of drugs as confounding factors on CRP levels. Also, the severity of the stroke could influence the level of CRP and so may have an influence on fatigue. A larger study would need to take into account the relationship between inflammation, fatigue and stroke severity. Finally, CRP is only one measure of inflammation; future studies might also measure other inflammatory cytokines.

In conditions other than stroke, where there is a relationship between fatigue and inflammation, the mechanisms are thought to be complex. In cancerrelated fatigue, pro-inflammatory cytokines may cause the fatigue by signalling the central nervous system to induce sleep and other 'sickness behaviours'. ${ }^{5}$ It has been shown that higher levels of IL-6, a pro-inflammatory cytokine that is produced during inflammatory processes, causes a change in the pattern of sleep and increased levels of fatigue. ${ }^{5}$ Alterations in circulating levels of this and other pro-inflammatory cytokines have also been noted in fatigue relating to other conditions, such as depression $^{18}$ and chronic fatigue syndrome. ${ }^{19}$ The physiological role of pro-inflammatory cytokines in the development of fatigue is, however, very complicated and poorly understood. Fatigue is a complex multidimensional symptom which is likely to be influenced by a variety of factors, and therefore a single cause is unlikely.

The next step is to explore the association between CRP and fatigue in a much larger cohort of patients. The data from this pilot study have informed power calculations for such a larger cohort study. If larger studies do confirm a relationship between inflammation and fatigue after 
stroke, this might provide a target for treatment. Given that there is currently no effective treatment for fatigue after stroke, a better understanding of its aetiology is key to the development of new therapies. ${ }^{20,21}$

Acknowledgements Permission for the study was granted by the Lothian Research Ethics Committee and the Research and Development office of NHS Lothian. We would like to thank all the patients who took part

\section{REFERENCES}

I De Groot MH, Phillips SJ, Eskes GA. Fatigue associated with stroke and other neurologic conditions: implications for stroke rehabilitation. Arch Phys Med Rehabil 2003;84:17|4-20.doi:I0.1053/ S0003-9993(03)00346-0

2 Ingles JL, Eskes GA, Phillips SJ. Fatigue after stroke. Arch Phys Med Rehabil 1999; 80:I73-8. doi:I0.1016/S0003-9993(99)90II6-8

3 Harbison JA, Walsh S, Kenny RA. Hypertension and daytime hypotension found on ambulatory blood pressure is associated with fatigue following stroke and TIA. Q J Med 2009; 102:109-15.

4 Wichers M, Maes M. The psychoneuroimmuno-pathophysiology of cytokine-induced depression in humans. Int J Neuropsychopharmacol 2002; 5:375-88. doi:10.1017/SI46I|45702003 I03

5 Bower JE. Cancer-related fatigue: links with inflammation in cancer patients and survivors. Brain Behav Immun 2007; 21:863-7I. doi:10.1016/j.bbi.2007.03.013

6 Collado-Hidalgo A, Bower JE, Ganz PA et al. Inflammatory biomarkers for persistent fatigue in breast cancer survivors. Clin Cancer Res 2006; 12:2759-66. doi:I0.II58/I078-0432.CCR-052398

7 Schubert C, Hong S, Natarajan L et al. The association between fatigue and inflammatory marker levels in cancer patients: a quantitative review. Brain Behav Immun 2007; 21:413-27. doi:I0.1016/j.bbi.2006.II.004

8 Paddison JS, Booth RJ, Fuchs D et al. Peritoneal inflammation and fatigue experiences following colorectal surgery: a pilot study. Psychoneuroendocrinology 2008; 33:446-54. doi:10.1016/j. psyneuen.2007.12.0II

9 Heesen C, Nawrath L, Reich C et al. Fatigue in multiple sclerosis: an example of cytokine mediated sickness behaviour? J Neurol Neurosurg Psychiatry 2006; 77:34-9. doi:I 0.I I 36/jnnp.2005.065805

10 Idicula TT, Brogger J, Naess $\mathrm{H}$ et al. Admission C-reactive protein after acute ischemic stroke is associated with stroke severity and mortality: the Bergen stroke study. BMC Neurol 2009; 9:18. doi:10.1 I86/I47I-2377-9-18

II Muir KW, Weir CJ, Alwan W et al. C-reactive protein and outcome after ischemic stroke. Stroke 1999; 32:98I-5. in the study for their time and co-operation, and the staff on the stroke units at the Royal Infirmary of Edinburgh, Astley Ainslie Hospital, Edinburgh, and Liberton Hospital, Edinburgh, for assisting us with identifying suitable patients. The laboratory staff at the Royal Infirmary of Edinburgh performed the CRP assays and Dr Mike Crane, Clinical Biochemist at the Royal Infirmary of Edinburgh, provided details of the method for CRP testing.
12 Smith CJ, Emsley HC, Gavin CM et al. Peak plasma interleukin-6 and other peripheral markers of inflammation in the first week of ischaemic stroke correlate with brain infarct volume, stroke severity and long-term outcome. BMC Neurol 2004; 4:2-9. doi: I0.I I86/I47|-2377-4-2

I 3 Vila N, Castillo J, Davalos A et al. Proinflammatory cytokines and early neurological worsening in ischemic stroke. Stroke 2000; 31:2325-9.

I4 Lynch J, Mead G, Greig C et al. Fatigue after stroke: the development and evaluation of a case definition. J Psychosom Res 2007; 63:539-44. doi:I0.1016/j.jpsychores.2007.08.004

I5 Mead G, Lynch J, Greig C et al. Evaluation of fatigue scales in stroke patients. Stroke 2007; 38:2090-5. doi:I0.II6I/ STROKEAHA. I06.47894I

16 Michielsen HJ, De Vries J, Van Heck GL. Psychometric qualities of a brief self-rated fatigue measure: the fatigue assessment scale. J Psychosom Res 2003; 54:345-53. doi:10.1016/S0022-3999(02)00392-6

17 Zigmond AS, Snaith RP. The hospital anxiety and depression scale. Acta Psychiatr Scand 1 983;67:36 I-70. doi: I0. I I I I/j. I600-0447.1983. tb09716.x

18 Musselman DL, Miller AH, Porter MR et al. Higher than normal plasma interleukin-6 concentrations in cancer patients with depression: preliminary findings. Am J Psychiatry 200I; I58:1252-7. doi: I0.I I76/appi.ajp.I58.8.I252

19 Gaab J, Rohleder N, Heitz V et al. Stress-induced changes in LPSinduced pro-inflammatory cytokine production in chronic fatigue syndrome. Psychoneuroendocrinology 2005;30:188-98. doi:10.1016/j. psyneuen.2004.06.008

20 Staub F, Bogousslavsky J. Fatigue after stroke: a major but neglected issue. Cerebrovasc Dis 200 I; I2:75-8I. doi: I 0. I I59/000047685

2I McGeough E, Pollock A, Smith LN et al. Interventions for poststroke fatigue. Cochrane Database Syst Rev 2009; 3: CD007030. doi:I0.1002//465I858.CD007030.pub2 